\title{
HCV1b genome evolution under selective pressure of the cyclophilin inhibitor alisporivir during the DEB-025-HCV-203 phase II clinical trial
}

\author{
Lize Cuypers $^{\mathrm{a}, *}, 1$, Joke Snoeck ${ }^{\mathrm{a}, 1,2}$, Lien Kerremans ${ }^{\mathrm{a}, 3}$, Pieter Libin ${ }^{\mathrm{b}, \mathrm{a}}$, Raf Crabbé ${ }^{\mathrm{c}}$, Sonia Van Dooren ${ }^{\mathrm{a}, 4}$, \\ Grégoire Vuagniaux ${ }^{\mathrm{c}}$, Anne-Mieke Vandamme ${ }^{\mathrm{a}, \mathrm{d}}$ \\ ${ }^{a}$ KU Leuven - University of Leuven, Department of Microbiology and Immunology, Rega Institute for Medical Research, Clinical and Epidemiological Virology, \\ Minderbroedersstraat 10, 3000 Leuven, Belgium \\ b Artificial Intelligence Lab, Vrije Universiteit Brussel, Pleinlaan 2, 1050 Brussels, Belgium \\ ${ }^{c}$ Debiopharm International S.A., Che. Messidor 5-7, P.O. Box 5911, 1002 Lausanne, Switzerland \\ ${ }^{\mathrm{d}}$ Center for Global Health and Tropical Medicine, Unidade de Microbiologia, Instituto de Higiene e Medicina Tropical, Universidade Nova de Lisboa, Rua da Jungquiera 100, \\ 1349-008 Lisbon, Portugal
}

\section{A R T I C LE INFO}

Article history

Received 24 March 2016

Received in revised form 24 June 2016

Accepted 27 June 2016

Available online $\mathrm{xxx}$

Keywords:

Alisporivir

Cyclophilin inhibitor

Full-genome sequencing

Abbreviations: BL, baseline; BLQ, below limit of quantification; BN, Bayesian network; (c)DNA, (copy) deoxyribonucleic acid; CsA, cyclosporine A; DAA, direct-acting antiviral; $\mathrm{dN}$, non-synonymous nucleotide change; $\mathrm{dS}$, synonymous nucleotide change; $\mathrm{EC}_{50}$, half maximal effective concentration; EDTA, ethylenediaminetetraacetic acid; GT, genotype; GTR, generalized time reversible; HCV, hepatitis $\mathrm{C}$ virus; HIV, human immunodeficiency virus; HTA, host-targeting antiviral; HVR1, hypervariable region-1; ID, identification; IFN, interferon; IRRDR, interferon and ribavirin resistance-determining region; ISDR, interferon sensitivity determining region; IU/ml, international units per $\mathrm{ml}$; mcg, microgram; N, number; N.D., not determined; pegIFN- $\alpha 2 \mathrm{a}$, pegylated interferon-alpha2a; PCR, polymerase chain reaction; RNA, ribonucleic acid; RT, reverse transcriptase; SVR, sustained virological response; UTR, untranslated region; VL, viral load; WT, wild-type

* Corresponding author at: Rega Institute for Medical Research, Minderbroedersstraat 10, 3000 Leuven, Belgium.

Email addresses: lize.cuypers@kuleuven.be (L. Cuypers); joke.snoeck@nds.ox. ac.uk (J. Snoeck); lienkerremans@hotmail.com (L. Kerremans); pieter.libin@vub. ac.be (P. Libin); rcrabbe@debiopharm.com (R. Crabbé); sonia.vandooren@, uzbrussel.be (S. Van Dooren); gregoire.vuagniaux@debiopharm.com (G. Vuagniaux); annemie.vandamme@uzleuven.be (A-M Vandamme)

${ }^{1}$ Both authors contributed equally to this work.

${ }^{2}$ Currently at the Nuffield Department of Surgical Sciences, John Radcliffe Hospital, Headington, Oxford, OX3 9DU, USA.

${ }^{3}$ Currently at Multiplicom N.V., Galileilaan 18, 2845 Niel, Belgium.

${ }^{4}$ Currently at the Center for Medical Genetics, Research Cluster Reproduction, Genetics and Regenerative Medicine, Research Group Reproduction and Genetics, Vrije Universiteit Brussel, Universitair Ziekenhuis Brussel (UZ Brussel), Laarbeeklaan 103, 1090 Brussels, Belgium.

\section{Introduction}

Chronic hepatitis caused by the hepatitis $\mathrm{C}$ virus (HCV) remains a global health problem, as it is accompanied with high morbidity and progression to life-threatening liver diseases. Worldwide, 80 million people are currently chronically infected with this virus (Gower et al., 2014; Hanafiah et al., 2013), which manifests into seven genotypes (GTs) and $>50$ subtypes (Smith et al., 2014). The former standard treatment with pegylated interferon- $\alpha$ (pegIFN- $\alpha)$ and ribavirin (Hofmann and Zeuzem, 2011) has greatly improved the outcome of $\mathrm{HCV}$ infections, although its success varied according to the genotype, with the lowest sustained virological response (SVR) rates achieved in HCV genotype 1 infected patients (Munir et al., 2010).

As genotype 1 infections prevail worldwide, these low SVR rates resulted into the development of the direct acting antivirals (DAAs), which specifically target $\mathrm{HCV}$ proteins such as NS3, NS5A and NS5B. The first DAAs still needed to be administered in combination with pegIFN- $\alpha$ and ribavirin, since their success was hampered by the fast selection of viral drug resistant variants (Barnard et al., 2013; Chevaliez, 2011; Halfon and Locarnini, 2011; Pawlotsky, 2011; Sullivan et al., 2013), especially when administered as monotherapy. In order to tackle drug resistance development, combination therapy strategies with multiple DAAs were introduced (Gane et al., 2010; Zeuzem et al., 2012), consisting of regimens which no longer need to be combined with interferon, and which all reach SVR rates of $>90 \%$ in all HCV genotypes (Andreone et al., 2014; Jacobson et al., 2014; Kowdley et al., 2013; Lawitz et al., 2013; Nelson et al., 2015). 
Drug resistance

D320E

$\mathrm{HCV}$

A B S T R A C T

Major advances have revolutionized the HCV antiviral treatment field, with interferon-free combinations of direct-acting antivirals (DAAs) resulting into success rates of $>90 \%$ for all HCV genotypes. Nevertheless, viral eradication at a global level stills remains challenging, stimulating the continued search for new affordable pan-genotypic drugs. To overcome selection of drug resistant variants, targeting host proteins can be an attractive mechanism of action. Alisporivir (Debio 025 ) is a potent pan-genotypic host-targeting antiviral agent, acting on cyclophilin A, which is necessary for HCV replication. The efficacy and safety of three different oral doses of alisporivir in combination with pegylated interferon- $\alpha 2 \mathrm{a}$ given over a period of four weeks, was investigated in a randomized, double-blind and placebo-controlled phase IIa clinical trial, in 90 treatment-naïve subjects infected with chronic hepatitis C, wherefrom 58 HCV1b samples were selected for genetic sequencing purposes. Sequencing results were used to study the HCV genome for amino acid changes potentially related with selective pressure and resistance to alisporivir. By comparing baseline and on-treatment sequences, a large variation in proportion of amino acid changes was detected in all treatment arms. The NS5A variant D320E, which was previously identified during in vitro resistance selection and resulted in 3.6-fold reduced alisporivir susceptibility, emerged in two subjects in the alisporivir monotherapy arm. However, emergence of D320E appeared to be associated only with concurrent viral load rebound in one subject with $0.8 \log _{10} \mathrm{IU} / \mathrm{ml}$ increase in HCV RNA. In general, for all datasets, low numbers of positions under positive selective pressure were observed, with no significant differences between naïve and treated sequences. Additionally, incomplete sequence information for some of the 22 patients and the low number of individuals per treatment arm, is limiting the power to assess the association of alisporivir or interferon treatment with the observed amino acid changes.

(C) 2016 Published by Elsevier Ltd.

Nevertheless, viral eradication of $\mathrm{HCV}$ at a global level is still hampered due to remaining challenges, such as the lack of a preventive vaccine. The high number of undiagnosed and untreated patients should be decreased, and special attention is required to specific risk groups for which high incidence rates of re-infection following SVR were reported (De Vos and Kretzschmar, 2014; Hill et al., 2015). The costs associated with these potent regimens are high, making it unlikely that all HCV infected patients worldwide will get access to treatment. Additionally, as the first instance of transmission of a telaprevir resistant $\mathrm{HCV}$ has recently been described (Franco et al., 2014), the impact of drug resistance in the HCV antiviral treatment field is not fully uncovered, resulting into the need of continued search towards new pan-genotypic antiviral drugs, such as cyclophilin inhibitors (Baugh et al., 2013; Lee, 2013), especially in case of retreating patients who previously failed a regimen consisting of all three DAA classes.

Instead of disrupting the replication complex by the inhibition of viral proteins, targeting host proteins can be an attractive mechanism of action (Nag et al., 2012). Cyclophilin A is essential for the HCV replication cycle, as it interacts together with HCV non-structural proteins to initiate the formation of the replication complex, however whether or not a direct interaction is obliged, is not entirely understood. Its inhibitor cyclosporine A (CsA) is a powerful albeit toxic anti HCV drug (Buti et al., 2014; Chatterji et al., 2014; Griffel et al., 2012), as it inhibits the peptidyl prolyl-isomerase activity of cyclophilin A and blocks the phosphorylation of the NS5A protein by cyclophilin A. The non-immunosuppressive CsA derivative alisporivir (Debio 025) is a pan-genotypic HCV inhibitor (Crabbé et al., 2009; Flisiak et al., 2012; Gallay and Lin, 2013), of which potency was demonstrated in vitro (Coelmont et al., 2009; Paeshuyse et al., 2006), as well as in clinical settings, particularly in HCV3 infected subjects (Flisiak et al., 2008, 2009; Guedj et al., 2013; Pawlotsky et al., 2012). In a phase II study, alisporivir combined with pegIFN- $\alpha 2 \mathrm{a}$ and ribavirin achieved superior viral cure in $76 \%$ of the chronically HCV1 infected patients, in contrast to $55 \%$ in patients treated with pegIFN- $\alpha 2 \mathrm{a}$ and ribavirin alone (Flisiak et al., 2011).

Recently, alisporivir was explored as an interferon-free combination regimen with DAAs in HCV2 and 3 infected subjects, resulting into SVR rates ranging from $80 \%$ to $85 \%$ (Pawlotsky et al., 2015). Amino acid variants related to drug resistance have been reported for both drugs, alisporivir and pegIFN- $\alpha 2$ a. For alisporivir a limited number of reports are available, and they describe a few amino acid substitutions in the HCV genome, mainly in the NS5A region (Arai et al., 2014; Coelmont et al., 2010; Garcia-Rivera et al., 2012). With regard to pegIFN- $\alpha 2 \mathrm{a}$, the interferon sensitivity-determining region (ISDR) and the interferon ribavirin resistance-determining region (IRRDR), both in the NS5A protein, are well known to play a role in the resis- different oral doses of alisporivir in combination with pegIFN- $\alpha 2 \mathrm{a}$ was investigated in treatment-naïve subjects infected with HCV genotypes 1 to 4 , in a randomized, double-blind and placebo-controlled setting (DEB-025-HCV-203) over a time period of four weeks (Flisiak et al., 2009). From all four cohorts, the best viral response was obtained in subjects treated with 600 or $1000 \mathrm{mg}$ alisporivir combined with $180 \mathrm{mcg} /$ week pegIFN- $\alpha 2 \mathrm{a}$. In patients with genotypes 1 and 4 , the 600 - and 1000-mg combination treatments induced a continuous decrease in viral load that reached $-4.61 \pm 1.88$ and $-4.75 \pm 2.19 \log _{10} \mathrm{IU} / \mathrm{ml}$ at week 4 , respectively. In patients infected with HCV genotypes 2 and 3, HCV RNA levels at week 4 were reduced by $-5.91 \pm 1.11$ and $-5.89 \pm 0.43 \log _{10} \mathrm{IU} / \mathrm{ml}$, respectively, with the same treatment regimens. Twenty-five HCV1b infected subjects were selected for genetic sequencing, based on HCV viral load evolution during treatment and after end of therapy $(<2 \log \mathrm{IU} / \mathrm{ml}$ viral load decline). Plasma-associated virus of these subjects was sequenced with the Sanger approach and HCV evolution under treatment was investigated.

\section{Materials and methods}

\subsection{Design of the DEB-025-HCV-203 study}

The efficacy of alisporivir in combination with pegIFN- $\alpha 2 \mathrm{a}$ in treatment-naïve subjects was investigated in a multicenter, randomized, double-blind, placebo-controlled escalating dose-ranging phase IIa study over a time period of four weeks. In total, 90 subjects infected with HCV genotypes 1-4 were included, discriminating between 60 potential slow responders $(56 \mathrm{HCV} 1$ and $4 \mathrm{HCV} 4)$ and 30 potential fast responders ( $7 \mathrm{HCV} 2$ and $23 \mathrm{HCV} 3)$, as stratified in a 2:1 unbalanced ratio. All subjects were randomized into four cohorts: three double-blind, placebo-controlled cohorts of 24 subjects each (cohorts I, II, and III) and one open non-comparative cohort of 18 subjects (cohort IV), as specified in Fig. 1. Doses of 200, 600, and $1000 \mathrm{mg}$ / day alisporivir in combination with pegIFN- $\alpha 2$ a $180 \mu \mathrm{g} /$ week for four weeks were compared with monotherapy of either $1000 \mathrm{mg} /$ day alisporivir or $180 \mu \mathrm{g} /$ week pegIFN- $\alpha 2 \mathrm{a}$. More details on treatment regimen and viral load are available in Table 1 . The best viral response was obtained in cohorts II and III, with a mean drop in viral load of about $4 \log \mathrm{IU} / \mathrm{ml}$ in patients infected with HCV genotypes 1 and 4, and of $6 \log \mathrm{IU} / \mathrm{ml}$ in patients infected with HCV2 and HCV3, after four weeks of therapy (Flisiak et al., 2009).

\subsection{Selection of samples}

Based on treatment status, viral load and sample availability, 58 


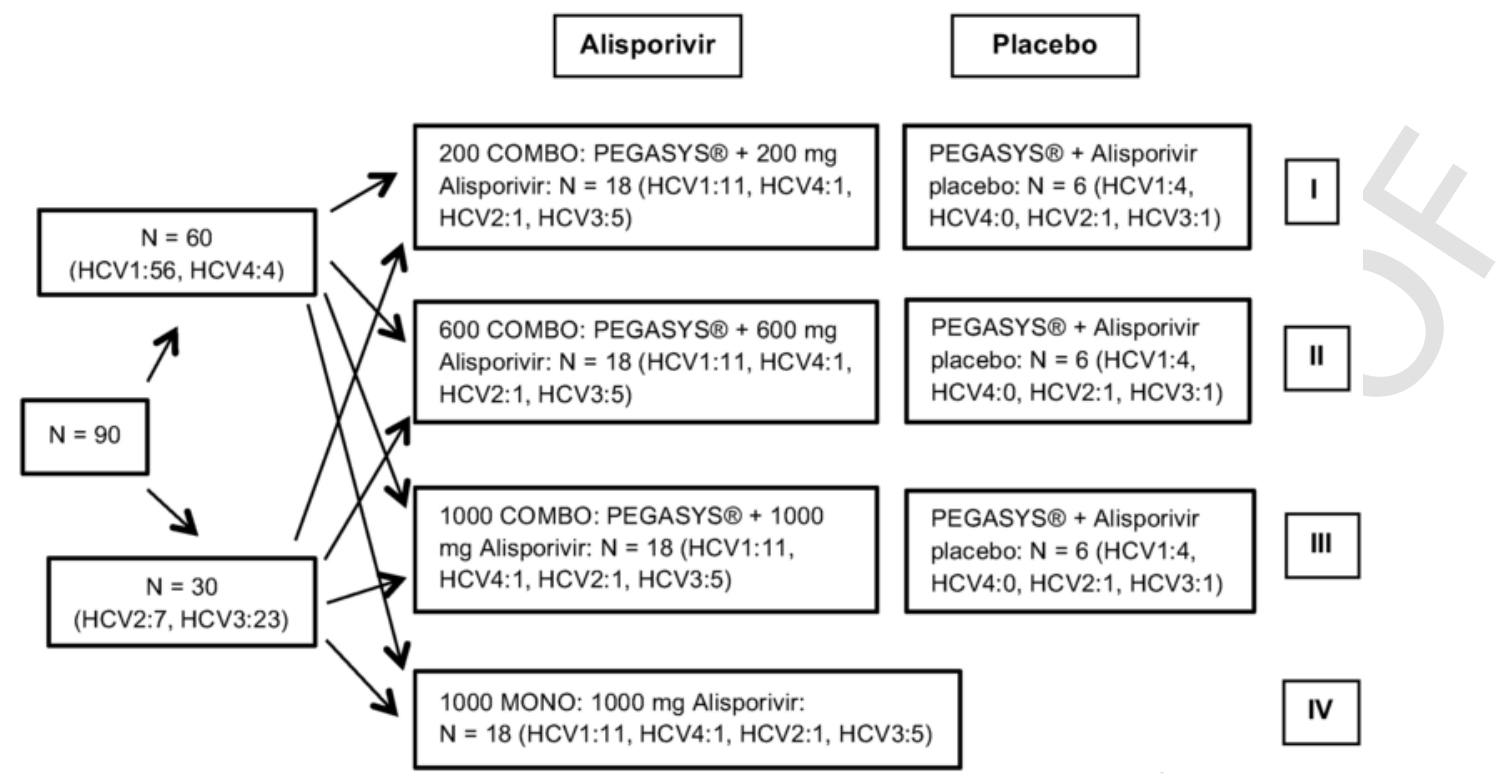

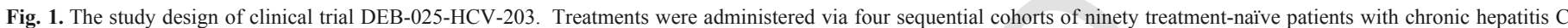

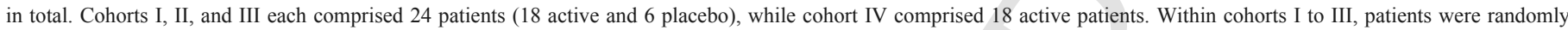

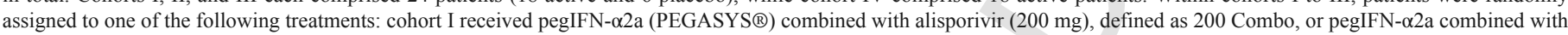

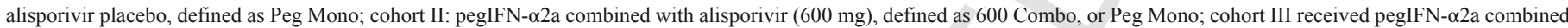

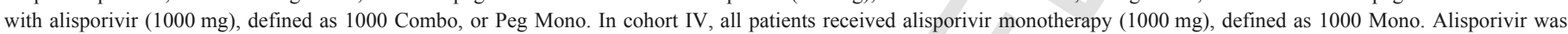

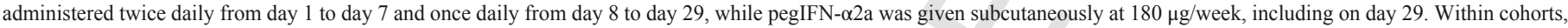

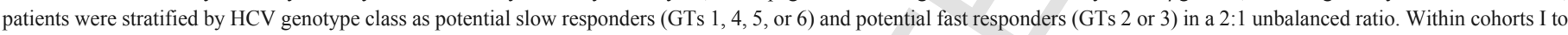

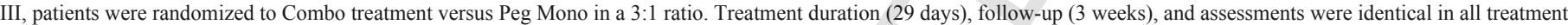
groups.

viral load response over a time period of 28 days, originating from the four different cohorts of the DEB-025-HCV-203 trial.

More in detail, all selected subjects are listed in Table 1: i) Twenty-one subjects with no or incomplete viral suppression at the end of the treatment period (Day 29) from all four cohorts; ii) Three subjects with complete viral suppression at Day 29 (viral load below limit of detection, $<15 \mathrm{IU} / \mathrm{ml}$ ), but detectable virus at the follow-up visit (Day 50) (>2 log IU/ml), from the 200 and 1000 Combo, and 1000 Mono treatment group (subjects 5202, 5501 and 1114; one from each group); and iii) One subject with detectable virus at Day 29, pegIFN- $\alpha 2$ a Mono group (subject 5409).

For all subjects, two samples were selected for genetic sequencing, more specifically a baseline sample (defined as the Day 1 sample) and an end-of-treatment sample (Day 29) or in some cases a follow-up sample (Day 50). A follow-up sample (Day 50) was only selected in case where the viral load of the sample at Day 29 was undetectable. Of two subjects in which the NS5A variant D320E was identified (subjects 5311 and 5415), three additional on-treatment samples (Day 8, 15 and 22) and one follow-up (Day 50) sample were analyzed, resulting into a total of 58 samples sequenced.

The viral load of all samples was determined using the HCV Viral Load COBAS AMPLICOR system, from Roche Molecular Diagnostics (Basel, Switzerland) and the HCV genotype using the Abbott RealTime HCV Genotype assay (Santa Clara, California). Both determinations were performed by MDS Pharma Services (Paris, France and North Brunswick, New Jersey). The viral loads of the samples varied from 35 to $5,919,998 \mathrm{IU} / \mathrm{ml}$. The majority of the samples were EDTA samples, for which our genetic sequencing protocols were optimized, however some were Li-heparin samples (12/58).

\subsection{Genotyping and genetic sequencing}

We use the term genotyping for assays performed with the sole purpose to determine to which genotype the HCV strains belong. The term genetic sequencing is used for assays performed to assess amino acid changes under treatment. The genotype of the samples was confirmed using a protocol based on sequencing a small region of the NS5B gene (Arrais et al., 2008). Once the HCV genotype was confirmed, viral sequences were determined by using a near-full genome HCV1b genetic sequencing method (Cuypers et al., 2014). Briefly, RNA was extracted using the QIAamp Viral RNA mini kit (Qiagen) and reverse transcribed with Transcriptor RT (Roche) in cDNA for three separate regions, 5'UTR-NS2, E2-NS5A and NS4B-NS5B, partially overlapping each other. Nested PCR was performed using the Expand Long Template PCR System (Roche) for the same three regions. The same (cycling) conditions were used in both PCRs. The achieved detection limit was $570 \mathrm{IU} / \mathrm{ml}$ for the 5'UTR-NS2 region, $5670 \mathrm{IU} / \mathrm{ml}$ for E2-NS5A, while for NS4B-NS5B a detection limit of $56,670 \mathrm{IU} / \mathrm{ml}$ was obtained.

\subsection{Sequencing and analyzing the sequences}

Sequencing reactions were performed at Fasteris SA (Geneva, Switzerland) using the BigDye Terminator v3.1 Cycle Sequencing kit (Applied Biosystems Inc., Foster City, USA). Afterwards contigs were generated and analyzed using SeqScape (Applied Biosystems Inc., Foster City, USA) and for each individual subject amino acid changes during treatment were reported with respect to the baseline sequence of that same subject. 


\subsection{Phylogenetic analysis}

Phylogenetic analysis was performed as part of the quality control, to confirm the genotype and to uncover potential sample mix-up. All NS5A-NS5B nucleotide sequences were aligned against four HCV1b reference sequences from the Los Alamos HCV Sequence Database (Kuiken et al., 2005), using an in-house developed pairwise alignment tool-chain (Cuypers et al., 2015; Libin, 2014), with the alignment manually edited using Seaview V4.0 (Gouy et al., 2010). A maximum-likelihood phylogenetic tree was constructed using RAxML (Stamatakis, 2014), with the GTR gamma model of substitution and 1000 bootstrap replicates to evaluate the robustness of the tree. The tree was visualized using FigTree v.1.4. (http://tree.bio.ed.ac.uk/ software/figtree/).

\subsection{Analysis of the amino acid variants}

In order to analyze the whole dataset, successfully amplified sequences were divided into four groups, according to treatment status with a specific drug: A) All baseline sequences (of all treatment arms) (group A, $\mathrm{n}=21$ ); B) All sequences after treatment of subjects receiving alisporivir monotherapy during four weeks (group $\mathrm{B}, \mathrm{n}=6$ ); $\mathrm{C}$ ) All sequences after treatment of subjects receiving alisporivir combined with pegIFN- $\alpha 2 \mathrm{a}$ during four weeks (group $\mathrm{C}, \mathrm{n}=12$ ); and D) One sequence after treatment (Day 29) of subject 5409 receiving weekly pegIFN- $\alpha 2$ a mono during four weeks, supplemented with four sequences from subjects not belonging to the DEB-025-HCV-203 clinical trial but after pegIFN- $\alpha 2$ a monotherapy for 24 weeks. Three of these HCV1b sequences were from Genbank (Enomoto et al., 1995) and one from an HCV1b chronically infected patient attending the University Hospital in Leuven, for which pegIFN- $\alpha 2$ a was combined with ribavirin (approved by the Leuven ethical committee: ML9219, subproject of ML8635) (group D, $\mathrm{n}=5$ ).

In a longitudinal analysis, amino acid changes were defined as the number of changes at amino acid level observed between the baseline (Day 1) and end-of-treatment sequence of the same subject, i.e. Day 29 (or follow-up sequence at Day 50 when viral load at Day 29 was too low) counting also indels and loss or gain of polymorphisms. Since not for all samples, the full-genome could be analyzed, due to some sequencing failures for parts of the genome, amino acid changes were also expressed in proportion. Standard linear regression was applied to study the association between the proportion of amino acid changes in the virus, and various measures of viral load for the subject in whom those changes were found: i) the drop in viral load (log change in viral load between baseline and Day 29), ii) the nadir viral load, iii) the viral load at baseline or iv) the viral load at Day 29. These correlations were explored based on the assumption that viral load is a possible measure for replication fitness, such that amino acid changes related to viral load during $\mathrm{HCV}$ antiviral treatment are possibly related to drug resistance.

In a cross-sectional analysis, Fisher's exact test was used to identify amino acid variants potentially associated with alisporivir- and interferon-treatment, located in proteins NS5A and NS5B (Libin, 2014). For assessing variants associated with alisporivir treatment, proportions of amino acid variants in baseline sequences (group A), and sequences after treatment with alisporivir (group B $+\mathrm{C}$ ) were compared; as well as group A with group B, the latter including only sequences under alisporivir monotherapy. For variants associated with interferon treatment, a similar comparison was made i) comparing baseline sequences (group A) with sequences after treatment with
pegIFN- $\alpha 2 \mathrm{a}$ (group $\mathrm{C}+\mathrm{D}$ ), and ii) focusing on pegIFN- $\alpha 2 \mathrm{a}$ monotherapy (group A vs group D). Amino acid variants with a significantly different proportion were used for Bayesian network (BN) learning to study possible associations with treatment, either with alisporivir or interferon, using an in-house developed tool chain, following the example of Deforche et al. (2006) and Deforche et al. (2008) and Libin, 2014 (see Supplements).

All sequences were also scanned for the presence of prolines and for variation around the proline sites in the genome, since cyclophilins are peptidyl-prolyl isomerases. As alisporivir may reduce the hyperphosphorylated form of NS5A (Quinkert et al., 2008), the presence of phosphorylation sites was checked, using the online available program NetPhos 2.0 (Blom et al., 1999). All sequences were compared to reference sequence HCV1bAJ238799 (Con1 strain).

\subsection{Selective pressure}

Detection of selective pressure was performed using the fixed effects likelihood (FEL) method, implemented in HyPhy v2.2.1 (Pond et al., 2005). The assumption that synonymous (dS) and non-synonymous $(\mathrm{dN})$ rates or $\mathrm{dN} / \mathrm{dS}$, vary among sites is premised, iterating through every codon position in the alignment to identify positions under significant positive or negative selective pressure (Pond et al., 2009). Positions characterized by a $\mathrm{dN} / \mathrm{dS}$ ratio $>1$ and a $\mathrm{p}$-value $<0.05$ were defined as positively selected sites, while positions with a $\mathrm{dN} / \mathrm{dS}$ ratio $<1$ and a $\mathrm{p}$-value $<0.05$ were considered to be under negative selective pressure. To evaluate the difference in $\mathrm{dN}$ and $\mathrm{dS}$ rates under alisporivir and interferon treatment, the same groups were compared as described in Section 2.6, using the two-tailed z-test to compare proportions between two groups, as implemented in Excel. Since only one sequence from a subject treated with IFN monotherapy was available, group D contained the four additional sequences from Genbank and from the University Hospital in Leuven as described under Section 2.6. An additional analysis was performed for subjects 5311 and 5415 harboring NS5A variant D320E, based on their NS5A-NS5B sequences during or after alisporivir treatment (Day 8, 15, 22, 29 and $50)$.

\section{Results}

\subsection{Amplification and sequencing}

From the 58 samples selected, only for 41 samples all three regions, 5'UTR-NS2, E2-NS5A and NS4B-NS5B could be amplified. For ten samples not all regions could be amplified, all ten had a low viral load (majority lower than $700 \mathrm{IU} / \mathrm{ml}$ ). For four samples none of the three regions could be amplified, for the first three samples probably due to their low viral loads $(<350 \mathrm{IU} / \mathrm{ml})$ and for the fourth sample, which had a higher viral load $(\sim 200,000 \mathrm{IU} / \mathrm{ml})$, amplification failure was probably because it was a Li-heparin sample which is known to inhibit subsequent PCR amplification. Of the follow-up samples taken at Day 50, all characterized by a very low viral load, for only one sample all regions could be amplified. As a result, for only 54 of the 58 samples full or partial genome sequences could be obtained. For the eight additional on-treatment samples of subjects 5415 and 5311, in whom the viral NS5A variant D320E was observed, region NS5A-NS5B was amplified successfully, with no attempt to amplify other regions. Successfully amplified regions were sequenced with the Sanger approach, of which $1.8 \%$ of runs did not result in good quality sequences, because of poor performance of some primers. Nevertheless there was sufficient information available 
for most regions, due to substantial overlap of sequenced fragments. More details can be found in Table 1.

\subsection{Samples excluded from analysis}

For four samples of two different subjects (subjects 3302 and 3305 ) from the 1000 Mono group, phylogenetic analysis revealed that probably a sample mix-up had occurred. For both subjects, the sample indicated as baseline did not cluster with the sample indicated as Day 29 of the corresponding subject, but with the samples of the other subject. Sequencing five more samples of both subjects (screening visit (before Day 1), Day 8, 15, 22 and 50) confirmed the switching between both baseline samples of subject 3305 and 3302 (Fig. S1). The occurrence of a transmission chain is less likely than a sample mix-up, since the other samples of the same subject did cluster together. Additionally, a third subject (3111) was excluded from the analysis, due to the detection of two subtypes in its baseline sample: four sequencing reactions indicated an infection with HCV1b, compared to all other reactions that pointed to HCV1a. In the sample taken at Day 29, only $\mathrm{HCV} 1 \mathrm{~b}$ virus was observed. Sequencing this baseline sample by using an HCV1a adapted protocol, confirmed that both viruses originated from the same sample, suggesting that this subject had a dual infection with subtypes HCV1a and $1 \mathrm{~b}$ (data not shown). Thus, in total six HCV genomes from three subjects were excluded from analysis (Table 2). This resulted in 48 partial or full-genome sequences (Table 1), remaining for further analyses. Sequences obtained in this study were submitted to Genbank (accession numbers KX258962-KX259009).

\subsection{Number of changes in amino acid sequence}

Amino acid sequence changes between the baseline and end-of-treatment or follow-up sample of the same subject were investigated for 48 samples. The viral load evolution of each subject is known over a time period of 28 days (Table 1), as well as the corresponding number of changes over (a part of) the full-genome (Table 2). Only the samples of two subjects showed no changes, originating from the 1000 Mono (subject 3308) and the 200 Combo (subject 5403 ) group. The largest proportion of changes found was $1.9 \%$ (16 changes for subject 5501 from the 1000 Combo group), but also for the samples of two subjects from the 1000 Mono group high proportions of $0.5 \%$ (15 changes for subject 1115 ) and $0.6 \%$ (19 changes for subject 5210) were identified. Most changes were loss or gain of polymorphisms. The median proportion of amino acid changes was compared for the four different cohorts, with respective proportions of $0.23 \%$ (IQR: $0.23 \%-0.37 \%), 0.35 \%(0.27 \%-0.45 \%), 0.37 \%$ $(0.27 \%-1.14 \%)$ and $0.23 \%(0.08 \%-0.47 \%)$ for cohort 200 Combo, 600 Combo, 1000 Combo and 1000 Mono. For the subject treated with pegIFN- $\alpha 2$ a monotherapy (5409), $0.6 \%$ or 18 changes were detected in the full-genome amino acid sequence, with half of them located in the NS5A-NS5B region. Notably, about half of all full-length sequences displayed a higher number of changes in this region (Table 2). No significant correlations were detected between the proportion of changes (neither full-genome, nor for the separate genes); and the viral load at baseline, the viral load at Day 29, the drop in viral load, or the nadir viral load.

Next, amino acid positions in NS5A-NS5B sequences significantly associated with therapy, were investigated (Table 3). When assessing variants under alisporivir treatment (see Materials and methods), the prevalence of amino acid variants at 52 positions was significantly different between naïve (group A) and treated (group B and C) subjects. Focusing only on subjects treated with alisporivir monotherapy (group A vs group B), revealed amino acid variants at 54 positions that had a significantly different prevalence, since this second cohort was characterized by amino acid variants at five additional positions, but missed three of the positions which were significant in the first cohort (Table 2). Under pegIFN- $\alpha 2$ a selective pressure, amino acid variants at 62 positions were significantly different (group A vs group $\mathrm{C}+\mathrm{D}$ ), with the majority of the positions being the same as those of the alisporivir comparison, potentially due to the extensive overlap in both treated subject groups (group C is present in both comparisons). When focusing on the five subjects treated with pegIFN- $\alpha 2 \mathrm{a}$ mono (group A vs group D), 56 significantly associated amino acid positions were found, most of which overlapped with the alisporivir comparisons. Since the majority of the significant variants are identical in all comparisons, it is not surprising that using $\mathrm{BN}$ learning, none of these amino acid variants were directly connected to the treatment node, neither for alisporivir, nor for pegIFN- $\alpha 2$ a (Figs. S2-S3).

A proline scanning of all sequences was performed to check if an increased variation was observed around the proline residues. No significant differences were detected between baseline and sequences after end of therapy, neither in the position of the proline, nor in the sequence variation around proline. Also, no changes were observed at phosphorylation sites (data not shown).

\subsection{The amino acid variant $D 320 E$}

Interestingly, the NS5A variant D320E, previously described as an amino acid variant contributing to 3.6 fold change in alisporivir potency during in vitro resistance selection with alisporivir (Coelmont et al., 2010), was identified in two subjects from the 1000 Mono treatment arm. For these two subjects, additional sequencing was performed for the NS5A-NS5B region in samples taken at Day 8, 15, 22 and 50 (Fig. 2). These sequences were of sufficient quality to assess whether $320 \mathrm{E}$ was a minority variant (represented by a quarter purple disk), present in more or less equal proportion with the wild type (half purple disk), or whether only 320E was detected (full purple disk). Subject 5311 did not respond virologically, although 320E appeared only around Day 22, constituting about half of the population at the end of treatment (54\% at Day 29), and became undetectable again off treatment. Subject 5415 had a slow $2 \log \mathrm{IU} / \mathrm{ml}$ drop in viral load by Day 22, but rebounded at Day 29 with a virus where 320D was completely replaced with $320 \mathrm{E}$, which persisted even off therapy. Standard regression analysis indicated that the presence of D320E was not significantly associated with change in viral load ( $\mathrm{p}$-value $=0.54)$, and viral load by itself was also unable to predict the presence of D320E $(\mathrm{p}$-value $=0.49)$.

\subsection{Selective pressure}

Using FEL, the number of positively and negatively selected positions was mapped for the full-genome sequences of the alisporivir and interferon datasets (Table 4). The number or proportion of sites under selective pressure was assessed, and these were compared between groups as indicated in methods. With regard to positive selective pressure, very few sites were selected, with the highest number found for the baseline dataset ( 14 sites or $0.46 \%$ ), however, there was no significant difference neither in the number nor proportion of sites when comparing subjects with or without drug selective pressure, whether alisporivir or pegIFN- $\alpha 2 \mathrm{a}$ in combination or monotherapy. Some of the positively selected sites were common in several comparisons. With regard to positions under negative selective pressure, the highest number was again observed for the baseline dataset (1282 


\section{Subject 5311}

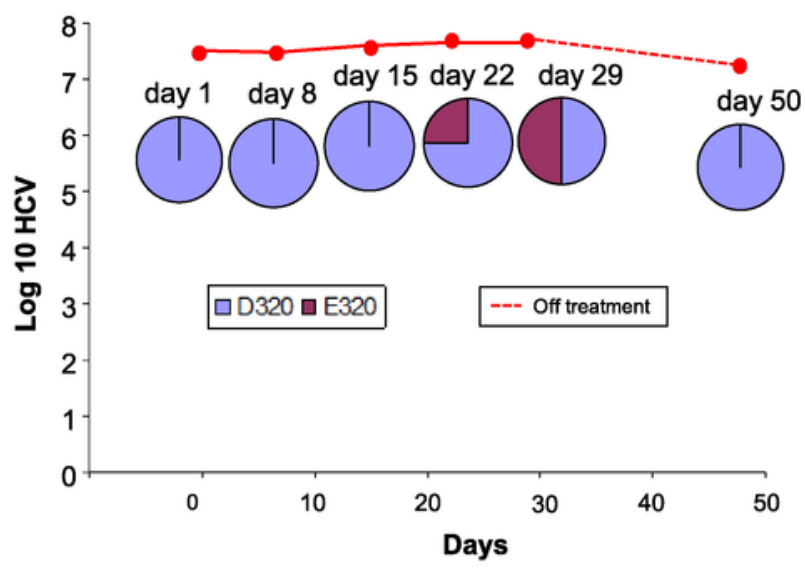

Subject 5415

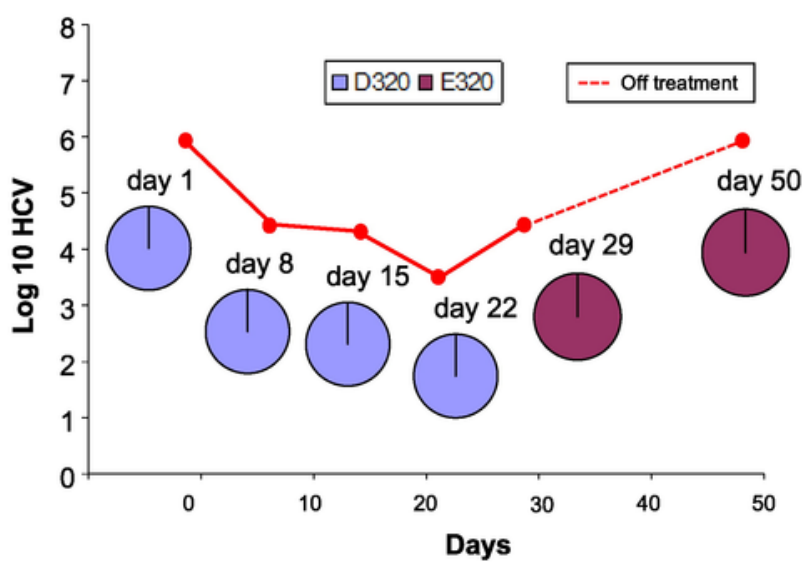

Fig. 2. Emergence of NS5A variant D320E in subjects 5311 and 5415. Subject 5311 did not show a virological response, its viral load remained stable and high, nevertheless there was a clear evolution at position 320 , representing about half of the viral population at Day 29. Subject 5415 showed a 2-log transient virological response, rebounding to pre-treatment levels when $320 \mathrm{E}$ emerged (320D could no longer be detected).

sites or $42.52 \%$ ), and again this was not significantly different in any of the comparisons. It has to be noted that the baseline dataset is the largest dataset $(\mathrm{N}=21)$, with the largest power to detect sites under positive or negative selection. Potential differences between datasets may be masked due to their small sample sizes.

Both in the alisporivir- and IFN-treated datasets, NS5A variant $320 \mathrm{D}$ was found to be under negative selective pressure. Additionally, $\mathrm{dN} / \mathrm{dS}$ rates were explored for the follow-up NS5A-NS5B sequences (Day 8, 15, 22 and 50) of the two subjects (5311 and 5415) harboring NS5A variant D320E, revealing no positive or negative selective pressure for NS5A position 320 .

\subsection{Other variants}

Five other variants were defined by Arai et al. (2014) as possibly conferring or enhancing resistance to CsA, more specifically T1280V in NS3, and D2292E, D2303H, S2363G and E2414K in NS5A. From these five mutations, variants T1280V (NS3) and D2292E (NS5A) occurred in the whole dataset with a respective prevalence of $1 \%$ and $4 \%$. Both resistance-related variants were not found to be under positive selective pressure. Subject 5411 (from the 1000 Combo group), with a virological response of $1.6 \mathrm{log}$, presented changes at amino acid positions previously reported during in vitro resistance selection (Table 2), more in detail I/V298V in NS5A (with V298A observed in vitro under alisporivir selective pressure, Neyts, 2008) and S556G in NS5B (Fernandes et al., 2007). However, there was no correlation between the presence of these variants, and the viral load change between baseline and Day 29, or the treatment group associated. Overall, the prevalence of both variants at baseline was $9 \%$, with none of them evolving under drug selective pressure.

\subsection{Resistance towards interferon}

Variants possibly related with resistance to interferon (Taylor et al., 2000; Perales et al., 2014) are located in the core region, the hypervariable region-1 (HVR-1) of protein E2, and in the ISDR (interferon sensitivity-determining region) and IRRDR (interferon and ribavirin resistance-determining region) of protein NS5A. In total, 70\% and $20 \%$ of all sequences in this study harbor the core variant $91 \mathrm{M}$ or 70Q respectively, both reported as associated with IFN treatment failure (Seko et al., 2013). Yet, no significant difference was observed between alisporivir mono (group B) and pegIFN- $\alpha 2$ a mono-treated (group D) subjects for the presence of both core variants ( $\mathrm{p}$-value $(70 \mathrm{Q}$ and $91 \mathrm{M})=0.49)$. For protein NS5A, sequence heterogeneity in the ISDR and IRRDR has been shown to be associated with a favorable response to IFN-based therapy (Enomoto et al., 1996; Hayashi et al., 2011; Kozuka et al., 2012; El-Shamy et al., 2011). Since only longitudinal data was available for one subject treated with interferon monotherapy, the number of amino acid changes, present in both the ISDR and IRRDR of NS5A, as well as in the HVR-1 of protein E2, could not be compared between alisporivir- and IFN-treated subjects.

\section{Discussion}

To investigate the evolution of HCV during alisporivir treatment, $25 \mathrm{HCV} 1 \mathrm{~b}$ infected subjects from the DEB-025-HCV-203 phase II dose escalating study were studied, selecting subjects based on treatment arm allocation, viral load response (no or incomplete viral suppression over a time period of four weeks) and samples availability. In total, 58 samples were genotyped, using a near-full genome sequencing approach. For each subject, a baseline sample was compared with a sample taken at the end of the treatment period (Day 29) or at the follow-up visit (Day 50). For two subjects characterized by the presence of NS5A variant D320E, an amino acid variant known to confer low level resistance to alisporivir in vitro, additional on-treatment samples were sequenced. From the 25 subjects, three subjects were excluded from the analysis, due to the occurrence of a sample mix-up and a dual infection with HCV1a and HCV1b.

\subsection{Number of changes in amino acid sequence}

All subjects with available sequence information for both a baseline and Day 29 or Day 50 sample, were checked for amino acid changes in the full-genome or in the successfully amplified regions, during course of treatment. Since the viral dynamic considerably changes over an additional time period of 21 days, the comparative analysis might have been influenced by the selection of Day 50 samples for a limited number of subjects. Nevertheless, since HCV RNA levels were under the limit of detection for their respective Day 29 samples, and to avoid reducing the number of subjects even further, we decided to take those Day 50 samples in order to have information on amino acid changes for those subjects.

Although a large variation in the proportion of sequence changes was detected, no correlation could be found between the proportion 
of changes; and the drop in viral load, the nadir viral load, the viral load at baseline and Day 29, or the treatment arm the subject was included in, similar to observations in a previous study (Welker et al., 2007). Nevertheless, for the ISDR of the NS5A protein, associations between amino acid changes and viral load levels, have been reported (Kobayashi et al., 2002; Watanabe et al., 2003). Using a Fisher's exact test, amino acid variants with significant differences in prevalence between baseline and post-treatment, in subjects either treated with alisporivir and/or pegIFN- $\alpha 2 \mathrm{a}$, were selected. The majority of the amino acid positions associated with treatment overlapped between alisporivir and pegIFN- $\alpha 2 \mathrm{a}$, and this may be related to the fact that they both elicit resistance within NS5A, despite having a different mechanism of action (Coelmont et al., 2010; Kozuka et al., 2012). In addition, in an association study through $\mathrm{BN}$ learning, none of the variants could be linked to either alisporivir or pegIFN- $\alpha 2 \mathrm{a}$. However the interpretation of these results is complicated, due to the extensive overlap in subjects assigned to the alisporivir- or IFN-treated groups $(\mathrm{N}=12)$, as well as the low number of subjects treated with only one of the two drugs in monotherapy. Only one mono pegIFN- $\alpha 2 a$-treated subject was selected for sequencing, and the IFN monotherapy group was therefore expanded with three additional subjects whose sequences were deposited in Genbank, and one extra subject from a different cohort (Leuven University Hospital). However, since the mono-treated groups did show many of the same amino acid positions associated with treatment, this would suggest they may rather play a role in replication fitness. Other hypotheses should also be explored, such as random genetic drift. To better assess their impact on treatment or fitness, higher numbers of sequences from treated subjects are needed. The use of Bayesian network learning in the context of HIV drug resistance has proven to be able to uncover resistance-related amino acid changes (Deforche et al., 2008).

\subsection{Selective pressure}

Using FEL implemented in HyPhy, sites under significant positive or negative selective pressure could be identified in subjects treated with alisporivir and pegIFN- $\alpha 2$ a combined or monotherapy. Although the number of such sites varied, there was no significant difference between treatment regimens, and positively selected sites were few $(0.07$ to $0.46 \%)$, while a substantial proportion was under negative selective pressure (12.6 to $42.52 \%$ ). The most striking finding of this analysis was that despite the large number of changes under therapy, only very few sites can be identified as being under positive selective pressure, seemingly not related to therapy, and a large proportion of sites is under negative selective pressure even during therapy. This observation confirmed the results of former studies which also identified a low number of positively selected positions in the HCV genome (Cuypers et al., 2015; Gray et al., 2011; Sheridan et al., 2004; Suzuki and Gojobori, 2001; Thomson et al., 2011), especially compared to other viruses like HIV (12\% of sites under positive selective pressure) (Snoeck et al., 2011).

\subsection{NS5A variant D320E}

The first to identify the importance of NS5A variant D320E was Goto et al. (2009), who observed that the HCV replicon with this mutation exhibited reduced susceptibility to CsA, resulting into limited HCV RNA treatment response. This hypothesis was confirmed by Puyang et al. (2010) who selected two NIM811-resistant clones with six amino acid changes, and found that the level of resistance achieved by the single D320E variant was nearly the same as that seen with the NS5A gene containing all six mutations. Coelmont et al. (2010) performed an in vitro selection of low-level resistance to alisporivir, and found D320E in NS5A. The D320E variant produced a moderate 3.6 fold increase of $\mathrm{EC}_{50}$ for alisporivir in the $\mathrm{HCV}$ replicon (Flisiak et al., 2008).

In this study, variant D320E was identified in only two subjects (subjects 5415 and 5311) from the 1000 Mono treatment arm, while a third subject (5210) from the same arm has been reported to harbor D320E as a minority variant using clonal sequencing (7\%, Tiongyip et al., 2011). In subject 5415, the acquisition of D320E corresponded to a rebound in viral load by $0.8 \mathrm{log} \mathrm{IU} / \mathrm{ml}$ between Day 22 and Day 29 , after an initial decrease in viral load of $2 \log \mathrm{IU} / \mathrm{ml}$. However, in the case of subject 5311, the emergence of D320E did not appear to be associated with viral load changes, as well as for subject 5210 . Given the high level of conservation of site 320 and the appearance of $320 \mathrm{E}$ only in subjects treated with a high dose of alisporivir, it would seem that D320E is resulting from drug selective pressure. However, its positive selective pressure was not strong enough to be uncovered by our methods in this dataset. In fact, D320E was under negative selective pressure in the overall analysis, suggesting it is important for the virus to maintain D320. Yet, the persistence off therapy suggests that this variant does not have a dramatic fitness cost, or that compensatory mutations have accumulated. Indeed, the development of compensatory mutations for viral fitness loss due to resistance mutations has been suggested in HCV (Di Maio et al., 2015). Similar to DAAs, it is expected that the emergence of high level resistant strains to alisporivir would demonstrate a sudden loss of viral suppression. Statistical analysis indicated that the presence of D320E was not significantly associated with viral load change, and the latter was not predictive for the presence of this variant. Notably, in this study, D320E was detected in subjects with little or no viral load reduction over four weeks of treatment.

According to Garcia-Rivera et al. (2012), multiple mutations in the NS5A domain II are required to confer a significant level of resistance to alisporivir, for instance variant D320E combined with variant Y321N. However none of the HCV1b sequences in this study harbored Y321N. The additive effect of other mutations was also reported by Tiongyip et al. (2011), which identified an increase of 4.76 fold in $\mathrm{EC}_{50}$ for the entire mutant compared to only 2.65 fold for D320E alone. The rather low fold change in susceptibility to alisporivir $(<3$ fold change), resulting from phenotypic analyses for the subjects harboring variant D320E (Tiongyip et al., 2011), and the lack of association with a change in viral load, argues against viral resistance as the primary cause of poor response to alisporivir. Therefore, the importance of this amino acid change in alisporivir treatment still needs to be better documented. Additionally, it was reported that alisporivir-resistant clones including NS5A substitutions remained fully susceptible to all three DAA classes as well as that subjects bearing major DAA resistant variants to all three DAA classes can still be treated with alisporivir (Tiongyip et al., 2011).

\subsection{Other variants}

Arai et al. (2014) reported five other mutations possibly conferring or enhancing resistance to CsA, of which four were located in the NS5A protein and the fifth in the NS3 region. Only two of them were found in this study, more in detail the NS3 variant T1280V and variant D2292E in NS5A, detected in respectively $1 \%$ and $4 \%$ of all sequences. Further, none of the other viral changes found were previously reported as being associated with resistance against alisporivir (Kaul, 2008; Snoeck, 2009) or against other cyclophilin inhibitors in vitro (Fernandes et al., 2007; Hopkins et al., 2012; Puyang et al., 2010; Robida et al., 2007). Although a subject was detected with a 
virus that showed variation on previously described amino acid positions 298 in NS5A and 556 in NS5B, also here no statistical significant correlations were detected between changes at one of these positions and change in viral load.

\subsection{Resistance to interferon}

Several variants have been reported to be potentially related with resistance to IFN (Perales et al., 2014; Taylor et al., 2000), in the core protein variants $91 \mathrm{M}$ and 70Q (Seko et al., 2013), the hypervariable region-1 (HVR-1) of protein E2, and sequence variation in the ISDR (interferon sensitivity-determining region) and the IRRDR (interferon and ribavirin resistance-determining region) of protein NS5A (El-Shamy et al., 2011; Hayashi et al., 2011; Kozuka et al., 2012). Subject 5409, only treated with interferon, harbored 18 amino acid changes in the full-genome of HCV1b, of which nine were located in the NS5A-NS5B genes. One of these was 91M in the core protein, however, more than half of all studied subjects harbored this specific variant. However, no significant differences were detected when comparing subjects treated with alisporivir and/or interferon, which could be due to the low number of sequences under selective pressure with alisporivir or pegIFN- $\alpha 2$ a monotherapy.

\section{Conclusion}

The study DEB-025-203 was too small to make any statistically significant conclusions on amino acid changes that could be associated with virological response or failure. Interestingly, variant D320E in NS5A, previously detected during in vitro resistance selection, was identified in two subjects treated with alisporivir monotherapy, however associated with only a small increase in viral load in one subject, not found to be positively selected, and displaying only a limited phenotypic effect (Tiongyip et al., 2011). Although the majority of HCV patients treated with DAA combinations seem to eradicate the virus, these results indicate that host targeting antivirals can still be of importance in the cure of potential hard-to-treat HCV patient populations.

\section{Funding}

Lize Cuypers was supported by a $\mathrm{PhD}$ grant of the FWO (Fonds Wetenschappelijk Onderzoek - Vlaanderen, Asp/12) and Joke Snoeck by a postdoctoral grant of the FWO (PDOH/09/FWO). Part of this research was sponsored by two FWO grants (G.A029.11N and $1 \mathrm{~S} 31916 \mathrm{~N})$, by an OT grant (OT/08/047) from the Research Fund KU Leuven, FWO budget (1.5.252.12N), a grant provided by the VUB (VUB/OZR2714) and by Debiopharm SA. The computational resources and services used in this work were provided by the Hercules Foundation and the Flemish Government - department EWI-FWO Krediet aan Navorsers (Theys, KAN2012 1.5.249.12.). Grégoire Vuagniaux and Raf Crabbé are employed by Debiopharm. The authors declare that they have no other competing interests than the financial disclosures above.

\section{Uncited references}

Ashfaq et al., 2011

Inoue et al., 2007

Ptak et al., 2008

\section{Acknowledgements}

The authors wish to thank Kristof Theys for his help with the Bayesian network learning, and Kristel Van Laethem for her participation and useful input during several discussions. The authors are also thankful to Andrea-Clemencia Pineda-Peña for assistance in the phylogenetic analysis and to Carolina Alvarez for her help with the statistical analysis.

\section{Appendix A. Supplementary data}

Supplementary data to this article can be found online at http://dx. doi.org/10.1016/j.meegid.2016.06.050.

\section{References}

Andreone, P., Colombo, M.G., Enejosa, J.V., et al., 2014. ABT-450, ritonavir, ombitasvir, and dasabuvir achieves $97 \%$ and $100 \%$ sustained virologic response with or without ribavirin in treatment-experienced patients with HCV genotype $1 \mathrm{~b}$ infection. Gastroenterology 147, 359-365. http://dx.doi.org/10.1053/j.gastro.2014. 04.045 .

Arai, M., Tsukiyama-Kohara, K., Takagi, A., et al., 2014. Resistance to cyclosporin A derives from mutations in hepatitis $\mathrm{C}$ virus nonstructural proteins. Biochem. Biophys. Res. Commun. 448, 56-62. http://dx.doi.org/10.1016/j.bbrc.2014.04.053.

Arrais, T.C., Van Dooren, S., Vandamme, A.M., et al., 2008. Change in hepatitis C virus genotype in hemodialysis patients after end-of-treatment response to interferon monotherapy-relapse or re-infection?. J. Med. Virol. 80, 80-86. http://dx. doi.org/10.1002/jmv.21050.

Ashfaq, U.A., Javed, T., Rehman, S., et al., 2011. An overview of HCV molecular biology, replication and immune responses. Virol. J. 8, 161. http://dx.doi.org/10. $1186 / 1743-422 X-8-161$

Barnard, R.J.O., Howe, J.A., Ogert, R.A., et al., 2013. Analysis of boceprevir resistance associated amino acid variants (RAVs) in two phase 3 boceprevir clinical studies. Virology 444, 329-336. http://dx.doi.org/10.1016/j.virol.2013.06.029.

Baugh, J.M., Garcia-Rivera, J.A., Gallay, P.A., 2013. Host-targeting agents in the treatment of hepatitis C: a beginning of an end?. Antivir. Res. 100, 555-561. http:// dx.doi.org/10.1016/j.antiviral.2013.09.020.

Blom, N., Gammeltoft, S., Brunak, S., 1999. Sequence and structure-based prediction of eukaryotic protein phosphorylation sites. J. Mol. Biol. 294, 1351-1362. http:// dx.doi.org/10.1006/jmbi.1999.3310.

Buti, M., Flisiak, R., Kao, J.H., et al., 2014. Alisporivir with peginterferon/ribavirin in patients with chronic hepatitis $\mathrm{C}$ genotype 1 infection who failed to respond to or relapsed after prior interferon-based therapy: FUNDAMENTAL, a phase II trial. J. Viral Hepat. 22, 596-606. http://dx.doi.org/10.1111/jvh.12360.

Chatterji, U., Garcia-Rivera, J.A., Baugh, J., et al., 2014. The combination of alisporivir plus an NS5A inhibitor provides additive to synergistic anti-hepatitis C virus activity without detectable cross-resistance. Antimicrob. Agents Chemother. 58, 3327-3334. http://dx.doi.org/10.1128/AAC.00016-14.

Chevaliez, S., 2011. Antiviral activity of the new DAAs for the treatment of hepatitis C virus infection: virology and resistance. Clin. Res. Hepatol. Gastroenterol. 35, S46-S51. http://dx.doi.org/10.1016/S2210-7401.

Coelmont, L., Kaptein, S., Paeshuyse, J., et al., 2009. Debio 025, a cyclophilin binding molecule, is highly efficient in clearing hepatitis $\mathrm{C}$ virus (HCV) replicon-containing cells when used alone or in combination with specifically targeted antiviral therapy for HCV (STAT-C) inhibitors. Antimicrob. Agents Chemother. 53, 967-976. http://dx.doi.org/10.1128/AAC.00939-08.

Coelmont, L., Hanoulle, X., Chatterji, U., et al., 2010. DEB025 (alisporivir) inhibits hepatitis $\mathrm{C}$ virus replication by preventing a cyclophilin A induced cis-trans isomerisation in domain II of NS5A. PLoS One 5, e13687http://dx.doi.org/10.1371/ journal.pone. 0013687 .

Crabbé, R., Vuagniaux, G., Dumont, J.M., et al., 2009. An evaluation of the cyclophilin inhibitor Debio 025 and its potential as a treatment for chronic hepatitis C. Expert Opin. Investig. Drugs 18, 211-220. http://dx.doi.org/10.1517/ 13543780802651583.

Cuypers, L., Snoeck, J., Vrancken, B., et al., 2014. A near-full length genotypic assay for HCV1b. J. Virol. Methods 209, 126-135. http://dx.doi.org/10.1016/j.jviromet. 2014.09.009.

Cuypers, L., Li, G., Libin, P., Piampongsant, S., Vandamme, A.M., Theys, K., 2015. Genetic diversity and selective pressure in hepatitis C virus genotypes1-6: significance for direct-acting antiviral treatment and drug resistance. Viruses 7, 5018-5039. http://dx.doi.org/10.3390/v7092857.

De Vos, A.S., Kretzschmar, M.E.E., 2014. Benefitis of hepatitis C virus treatment: a balance of preventing onward transmission and re-infection. Math. Biosci. 258, 8-11. http://dx.doi.org/10.1016/j.mbs.2014.09.006. 
Deforche, K., Silander, T., Camacho, R., et al., 2006. Analysis of HIV-1 pol sequences using Bayesian networks: implications for drug resistance. Bioinformatics 22, 2975-2979. http://dx.doi.org/10.1093/bioinformatics/btl508.

Deforche, K., Camacho, R.J., Grossman, Z., et al., 2008. Bayesian network analyses of resistance pathways against efavirenz and nevirapine. AIDS 22, 2107-2115. http:/ dx.doi.org/10.1097/QAD.0b013e32830fe940.

Di Maio, V.C., Cento, V., Di Paolo, D., et al., 2015. Compensatory mutations in HCV NS5A/B coevolve in patients failing NS3 inhibitors. CROI, Seattle, Washington, February 23-26, 2015.

El-Shamy, A., Shoji, I., Saito, T., et al., 2011. Sequence heterogeneity of NS5A and core proteins of hepatitis $\mathrm{C}$ virus and virological responses to pegylated-interferon/ ribavirin combination therapy. Microbiol. Immunol. 55, 418-426. http://dx.doi. org/10.1111/j.1348-0421.2011.00331.x.

Enomoto, N., Sakuma, I., Asahina, Y., et al., 1995. Comparison of full-length sequences of interferon-sensitive and resistant hepatitis $C$ virus $1 \mathrm{~b}$. J. Clin. Invest. 96, 224-230. http://dx.doi.org/10.1172/JCI118025.

Enomoto, N., Sakuma, I., Asahina, Y., et al., 1996. Mutations in the non-structural protein NS5A gene and response to interferon in patients with chronic hepatitis $\mathrm{C}$ virus $1 \mathrm{~b}$ infection. N. Engl. J. Med. 334, 77-81. http://dx.doi.org/10.1056/ NEJM199601113340203.

Fernandes, F., Poole, D.S., Hoover, S., et al., 2007. Sensitivity of hepatitis C virus to cyclosporine A depends on nonstructural proteins NS5A and NS5B. Hepatology 46, 1026-1033. http://dx.doi.org/10.1002/hep.21809.

Flisiak, R., Horban, A., Gallay, P., et al., 2008. The cyclophilin inhibitor Debio-025 shows potent anti-hepatitis $\mathrm{C}$ effect in patients coinfected with hepatitis $\mathrm{C}$ and human immunodeficiency virus. Hepatology 47, 817-826. http://dx.doi.org/10.1002/ hep. 22131 .

Flisiak, R., Feinman, S.V., Jablkowski, M., et al., 2009. The cyclophilin inhibitor Debio 025 combined with PEG IFN $\alpha 2$ a significantly reduces viral load in treatment-naïve hepatitis C patients. Hepatology 49, 1460-1468. http://dx.doi.org/10. 1002/hep. 22835.

Flisiak, R., Pawlotsky, J.M., Crabbe, R., et al., 2011. Once-daily alisporivir (DEB025) plus pegifnalfa2a/ribavirin results in superior sustained virological response (SVR24) in chronic hepatitis C genotype 1 treatment naïve patients. In: Presented at European Association for Study of the Liver Congress, Berlin, March 2011

Flisiak, R., Jaroszewicz, J., Flisiak, I., et al., 2012. Update on alisporivir in treatment of viral hepatitis C. Expert Opin. Investig. Drugs 21, 375-382. http://dx.doi.org/10. 1517/13543784.2012.658641.

Franco, S., Tural, C., Nevot, M., et al., 2014. Detection of a sexually transmitted hepatitis $\mathrm{C}$ virus protease inhibitor-resistance variant in a human immunodeficiency virus-infected homosexual man. Gastroenterology 174, 599-601. http://dx.doi.org 10.1053/j.gastro.2014.05.010.

Gallay, P.A., Lin, K., 2013. Profile of alisporivir and its potential in the treatment of hepatitis C. Drug Des. Devel. Ther. 7, 105-115. http://dx.doi.org/10.2147/DDDT S30946.

Gane, E.J., Roberts, S.K., Stedman, C.A., et al., 2010. Oral combination therapy with nucleoside polymerase inhibitor (RG7128) and danoprevir for chronic hepatitis C genotype 1 infection (INFORM-1): a randomised, double-blind, placebo-controlled, dose-escalation trial. Lancet 376, 1467-1475. http://dx.doi.org/10.1016/ S0140-6736(10)61384-0.

Garcia-Rivera, J.A., Bobardt, M., Chatterji, U., et al., 2012. Multiple mutations in hepatitis C virus NS5A domain II are required to confer a significant level of resistance to alisporivir. Antimicrob. Agents Chemother. 56, 5113-5121. http://dx.doi. org/10.1128/AAC.00919-12.

Goto, K., Watashi, K., Inoue, D., et al., 2009. Identification of cellular and viral factor related to anti-hepatitis $\mathrm{C}$ virus activity of cyclophilin inhibitor. Cancer Sci. 100, 1943-1950. http://dx.doi.org/10.1111/j.1349-7006.2009.01263.x.

Gouy, M., Guindon, S., Gascuel, O., 2010. SeaView version 4: a multiplatform graphical user interface for sequence alignment and phylogenetic tree building. Mol. Biol. Evol. 27, 221-224. http://dx.doi.org/10.1093/molbev/msp259.

Gower, E., Estes, C., Blach, S., et al., 2014. Global epidemiology and genotype distribution of the hepatitis C virus infection. J. Hepatol. 61, S45-S57. http://dx.doi.org/ 10.1016/j.jhep.2014.07.027.

Gray, R.R., Parker, J., Lemey, P., et al., 2011. The mode and tempo of hepatitis C virus evolution within and among hosts. BMC Evol. Biol. 11, http://dx.doi.org/10.1186/ 1471-2148-11-131.

Griffel, L., Bao, W., Orsenigo, R., et al., 2012. Alisporivir (DEB025) treatment in the VITAL-1 study has a more beneficial overall safety profile vs IFN-containing treatment. Hepatology 56, 792A.

Guedj, J., Yu, J., Levi, M., et al., 2013. Modeling viral kinetics and treatment outcome during alisporivir interferon-free treatment in hepatitis $\mathrm{C}$ virus genotype 2 and 3 patients. Hepatology 59, 1706-1714. http://dx.doi.org/10.1002/hep.26989.

Halfon, P., Locarnini, S., 2011. Hepatitis C virus resistance to protease inhibitors. J. Hepatol. 55, 192-206. http://dx.doi.org/10.1016/j.jhep.2011.01.011.

Hanafiah, M.K., Groeger, J., Flaxman, A.D., et al., 2013. Global epidemiology of hepatitis $\mathrm{C}$ virus infection: new estimates of age-specific antibody to HCV seroprevalence. Hepatology 57, 1333-1342. http://dx.doi.org/10.1002/hep.26141.

Hayashi, K., Katano, Y., Honda, T., et al., 2011. Association of interleukin 28B and mutations in the core and the NS5A region of hepatitis $\mathrm{C}$ virus with response to peg-interferon and ribavirin therapy. Liver Int. 31, 1359-1365. http://dx.doi.org/ 10.1111/j.1478-3231.2011.02571.x

Hill, A., Simmons, B., Saleem, J., et al., 2015. Five-year risk of late relapse or reinfection with hepatitis $\mathrm{C}$ after sustained virological response: meta-analysis of 49 studies in 8534 patients. In: 22nd CROI, Seattle, WA, February 23-26, 2015. Abstract 654

Hofmann, W.P., Zeuzem, S., 2011. A new standard of care for the treatment of chronic HCV infection. Nat. Rev. Gastroenterol. Hepatol. 8, 257-264. http://dx.doi.org/10. 1038/nrgastro.2011.49

Hopkins, S., Bobardt, M., Chatterji, U., et al., 2012. The cyclophilin inhibitor SCY-635 disrupts hepatitis C virus NS5A-cyclophilin A complexes. Antimicrob. Agents Chemother. 56, 3888-3897. http://dx.doi.org/10.1128/AAC.00693-12.

Inoue, K., Umehara, T., Ruegg, U.T., et al., 2007. Evaluation of a cyclophilin inhibitor in hepatitis C virus-infected chimeric mice in vivo. Hepatology 45, 921-928. http: //dx.doi.org/10.1002/hep.21587.

Jacobson, I.M., Dore, G.J., Foster, G.R., et al., 2014. Simeprevir with pegylated interferon alfa 2 a plus ribavirin in treatment-naïve patients with chronic hepatitis $\mathrm{C}$ virus genotype 1 infection (QUEST-1): a phase 3, randomised, double-blind, placebo-controlled trial. Lancet 384, 403-413. http://dx.doi.org/10.1016/ S0140-6736(14)60494-3.

Kaul, A., 2008. Further analyses of the antiviral effects of Debio 025 and understanding its mode of action. In: Study Debio-025-0135. Final Report.

Kobayashi, M., Watanabe, K., Ishigami, M., et al., 2002. Amino acid substitutions in the nonstructural region $5 \mathrm{~A}$ of hepatitis $\mathrm{C}$ virus genotypes $2 \mathrm{a}$ and $2 \mathrm{~b}$ and its relation to viral load and response to interferon. Am. J. Gastroenterol. 97, 988-998. http://dx.doi.org/10.1111/j.1572-0241.2002.05619.x.

Kowdley, K.V., Lawitz, E., Crespo, I., et al., 2013. Sofosbuvir with pegylated interferon alpha-2a and ribavirin for treatment-naïve patients with hepatitis $\mathrm{C}$ genotype-1 infection (ATOMIC): an open-label, randomized, multicentre phase 2 trial Lancet 381, 2100-2107. http://dx.doi.org/10.1016/S0140-6736(13)60247-0.

Kozuka, R., Enomoto, M., Hai, H., et al., 2012. Changes in sequences of core region, interferon sensitivity-determining region and interferon and ribavirin resistance-determining region of hepatitis $\mathrm{C}$ virus genotype 1 during interferon-alpha and ribavirin therapy, and efficacy of retreatment. Hepatol. Res. 42, 1157-1167. http://dx.doi.org/10.1111/j.1872-034X.2012.01046.x.

Kuiken, C., Yusim, K., Boykin, L., Richardson, R., 2005. The Los Alamos hepatitis C sequence database. Bioinformatics 21, 379-384. http://dx.doi.org/10.1093/ bioinformatics/bth 485

Lawitz, E., Mangia, A., Wyles, D., et al., 2013. Sofosbuvir for previously untreated chronic hepatitis C infection. N. Engl. J. Med. 368, 1878-1887. http://dx.doi.org/ 10.1056/NEJMoa1214853.

Lee, J., 2013. Cyclophilin A as a new therapeutic target for hepatitis C virus-induced hepatocellular carcinoma. Kor. J. Physiol. Pharmacol. 17, 375-383. http://dx.doi org/10.4196/kjpp.2013.17.5.375.

Libin, P., 2014. Applying graphical modelling techniques to virological data. In: The sis for the fulfilment of Master of Science in Applied Informatics. Vrije Universiteit Brussel. June 14.

Munir, S., Saleem, S., Idrees, M., et al., 2010. Hepatitis C treatment: current and future perspectives. Virol. J. 7, 296. http://dx.doi.org/10.1186/1743-422X-7-296.

Nag, A., Robotham, J.M., Tang, H., 2012. Suppression of viral RNA binding and the assembly of infectious hepatitis $\mathrm{C}$ virus particles in vitro by cyclophilin inhibitors. J. Virol. 86, 12616-12624. http://dx.doi.org/10.1128/JVI.01351-12.

Nelson, D.R., Cooper, J.N., Lalezari, J.P., et al. ALLY-3 Study Team, 2015. All-oral 12 -week treatment with daclatasvir plus sofosbuvir in patients with hepatitis C virus genotype 3 infection: ALLY-3 phase III study. Hepatology 61, 1127-1135. http://dx.doi.org/10.1002/hep.27726

Neyts, J., 2008. Characteristics of the anti-HCV activity of Debio 025. In: Study Debio 025-0141. Rega Institute. Updated report.

Paeshuyse, J., Kaul, A., De Clercq, E., et al., 2006. The non-immunosuppressive cyclosporin DEBIO- 025 is a potent inhibitor of hepatitis $\mathrm{C}$ virus replication in vitro. Hepatology 43, 761-770. http://dx.doi.org/10.1002/hep.21102.

Pawlotsky, J.M., 2011. Treatment failure and resistance with direct-acting antiviral drugs against hepatitis C virus. Hepatology 53, 1742-1751. http://dx.doi.org/10. 1002/hep.24262

Pawlotsky, J.M., Foster, G.R., Peng, C.Y., et al., 2012. Alisporivir plus ribavirin achieves high rates of sustained HCV clearance (SVR24) as interferon (IFN)-free or IFN-add-on regimen in treatment-naïve patients with HCV GT2 or GT3: final results from VITAL-1 study. Hepatology 56, 309A

Pawlotsky, J.M., Flisiak, R., Sarin, S.K., et al. on behalf of the VITAL-1 study team, 2015. Alisporivir plus ribavirin, interferon free or in combination with pegylated interferon, for hepatitis $\mathrm{C}$ virus genotype 2 or 3 infection. Hepatology 62, 1013-1023. http://dx.doi.org/10.1002/hep.27960.

Perales, C., Beach, N.M., Sheldon, J., et al., 2014. Molecular basis of interferon resistance in hepatitis C virus. Curr. Opin. Virol. 8, 38-44. http://dx.doi.org/10.1016/j. coviro.2014.05.003.

Pond, S.L., Frost, S.D., Muse, S.V., 2005. HyPhy: hypothesis testing using phylogenies. Bioinformatics 21, 676-679. http://dx.doi.org/10.1093/bioinformatics/bti079.

Pond, S.L., Poon, A.F.Y., Frost, S.D.W., 2009. Chapter 14: Estimating selection pressures on alignments of codon sequences: practice. In: P., L., M., S., A.M., V. 
(Eds.), The Phylogenetic Handbook: a Practical Approach to Phylogenetic Analysis and Hypothesis Testing, second ed. Cambridge University Press, New York, NY, USA, pp. 477-478.

Ptak, R.G., Gallay, P.A., Jochmans, D., et al., 2008. Inhibition of human immunodeficiency virus type 1 replication in human cells by Debio-025, a novel cyclophilin binding agent. Antimicrob. Agents Chemother. 52, 1302-1317. http://dx.doi.org/ 10.1128/AAC.01324-07.

Puyang, X., Poulin, D.L., Mathy, J.E., et al., 2010. Mechanism of resistance of hepatitis c virus replicons to structurally distinct cyclophilin inhibitors. Antimicrob. Agents Chemother. 54, 1981-1987. http://dx.doi.org/10.1128/AAC.01236-09.

Quinkert, D., Deneka, M., Najarro, P., et al., 2008. HCV inhibitors targeting NS5A. In: 15 th International Symposium on Hepatitis C Virus and Related Viruses, San Antonio, Texas, USA. abstract 302 .

Robida, J.M., Nelson, H.B., Liu, Z., et al., 2007. Characterization of hepatitis C virus subgenomic replicon resistance to cyclosporine in vitro. J. Virol. 81, 5829-5840. http://dx.doi.org/10.1128/JVI.02524-06.

Seko, Y., Akuta, N., Suzuki, F., et al., 2013. Amino acid substitutions in the hepatitis C virus core region and lipid metabolism are associated with hepatocarcinogenesis in nonresponders to interferon plus ribavirin combination therapy. Intervirology 56, 13-21. http://dx.doi.org/10.1159/000339993.

Sheridan, I., Phybus, O.G., Holmes, E.C., et al., 2004. High-resolution phylogenetic analysis of hepatitis $\mathrm{C}$ virus adaptation and its relationship to disease progression. J. Virol. 78, 3447-3454. http://dx.doi.org/10.1128/JVI.78.7.3447-3454.2004.

Smith, D.B., Bukh, J., Kuiken, C., et al., 2014. Expanded classification of hepatitis C virus into 7 genotypes and 67 subtypes: updated criteria and genotype assignment web resource. Hepatology 59, 318-327. http://dx.doi.org/10.1002/hep.26744.

Snoeck, J., 2009. Development of a near-full genome genotypic resistance assay for HCV genotype lb. In: Study Debio 025-0142. Final Report. Rega Institute.

Snoeck, J., Fellay, J., Barta, I., et al., 2011. Mapping of positive selection sites in the HIV-1 genome in the context of RNA and protein structural constraints. Retrovirology 8, 87. http://dx.doi.org/10.1186/1742-4690-8-87.
Stamatakis, A., 2014. RAxML version 8: a tool for phylogenetic analysis and post-analysis of large phylogenies. Bioinformatics 30, 1312-1313. http://dx.doi. org/10.1093/bioinformatics/btu033.

Sullivan, J.C., De Meyer, S., Bartels, D.J., et al., 2013. Evolution of treatment-emergent resistant variants in telaprevir phase 3 clinical trials. Clin. Infect. Dis. 57, 221-229. http://dx.doi.org/10.1093/cid/cit226.

Suzuki, Y., Gojobori, T., 2001. Positively selected amino acid sites in the entire coding region of hepatitis $\mathrm{C}$ virus subtype $1 \mathrm{~b}$. Gene 279 , 83-87. http://dx.doi.org/10.1016/ S0378-1119(01)00640-0.

Taylor, D.R., Shi, S.T., Lai, M.M., 2000. Hepatitis C virus and interferon resistance. Microbes Infect. 2, 1743-1756. http://dx.doi.org/10.1016/S1286-4579(00)01329-0.

Thomson, E.C., Smith, J.A., Klenerman, P., 2011. The natural history of early hepatitis $\mathrm{C}$ virus evolution; lessons from a global outbreak in human immunodeficiency virus-1-infected individuals. J. Gen. Virol. 92, 2227-2236. http://dx.doi.org/10 1099/vir.0.033910-0.

Tiongyip, C., Jones, C.T., Tang, Y., et al., 2011. Host targeting cyclophilin inhibitor alisporivir presents a high barrier to resistance with no cross-resistance to direct acting antivirals. In: Presented at the 6th International Workshop on Hepatitis C, Resistance and New Compounds, Cambridge, MA, June 24th, 2011.

Watanabe, H., Nagayama, K., Enomoto, N., et al., 2003. Sequence elements correlating with circulating viral load in genotype $1 \mathrm{~b}$ hepatitis $\mathrm{C}$ virus infection. Virology 311, 376-383. http://dx.doi.org/10.1016/S0042-6822(03)00155-7.

Welker, M.-W., Hofmann, W.-P., Welsch, C., et al., 2007. Correlation of amino acid variations within nonstructural $4 \mathrm{~B}$ protein with initial viral kinetics during interferon-alpha-based therapy in HCV-1b-infected patients. J. Viral Hepat. 14, 338-349. http://dx.doi.org/10.1111/j.1365-2893.2006.00798.x

Zeuzem, S., Buggisch, P., Agarwal, K., et al., 2012. The protease inhibitor, GS-9256, and non-nucleoside polymerase inhibitor tegobuvir alone, with ribavirin, or pegylated interferon plus ribavirin in hepatitis C. Hepatology 55, 749-758. http://dx. doi.org/10.1002/hep. 24744 . 